\title{
GEOMORPHOLOGY, CHARACTERISTICS AND MINERALOGY OF SOME SOILS IN EL-FARAFRA OASIS, NEW VALLEY GOVERNORATE, EGYPT
}

\author{
M. M. Soliman, Y. K. El Ghonamey and Shimaa K. Ganzour \\ Soils, Water and Environmental Research Institute (SWERI), ARC, Egypt. \\ E-mail: mahmoudaldemelawi@hotmail.com
}

Received: Sep. 17, 2019

Accepted: Nov. 3, 2019

ABSTRACT: The studied area belongs to El-Farafra Oasis, New Valley Governorate. It is representing an area of about 372062 feddans. The aim of this work was to study the geomorphology, characteristics and mineralogy of some soils in El-Farafra Oasis, New Valley governorate, Egypt.

Visual interpretation of 2 sentinel images and digital elevation model (DEM) were used to produce the geomorphic units and soil maps of the studied area. Two geomorphic units were identified namely plateau and depression floor. Eighty-two minipits and eighteen soil profiles were chosen to represent the main soil mapping units in the studied area. Soil samples were collected from the representative soil profiles for physical, chemical and mineralogical analysis. The results could be summarized as follows:

$X$-ray diffraction analysis indicated that kaolinite is alternatively dominated in the studied soils (about $80 \%$ ) followed by smectite. Illite, interstratified minerals and chlorite are found as the lowest abundant clay minerals in these soils.

The mineralogical composition of the sand fraction indicated that, the light fraction is composed almost entirely of quartz which constitutes about 95\%. Feldspar minerals (orthoclase plagioclase and microcline) are detected in trace amounts. The presence of feldspars indicates that, the soils are young from the pedological point of views.

Heavy minerals are generally dominated by opaque's (about $55 \%$ ). Non-opaques are mainly dominated by pyroboles (pyroxenes + amphipoles) followed by ultra-stable minerals (zircon, rutile and tourmaline), Para metamorphic minerals (garnet, kyanite, staurolite, silimanite) and epidote. While the other minerals (biotite andalusite, glaucohite and apatite) are detected in less pronounced amounts.

Distribution of the resistant minerals (biotite and amphipoles) weathering ratio, values indicate that, the soils are generally heterogeneous either due to their multi-origin or due to a subsequent variation along the course formation.

Key words: Geomorphic unit, Digital Elevation Model (DEM), clay mineralogy, Soil homogeneity, RS, GIS

\section{INTRODUCTION}

El- Farafra oasis is one of the main five Oases of the Western Desert, with an excellent groundwater resources and potential soils for agricultural expansion. Thus it considered as promising for future agriculture and tourist development. It is the second largest depression in size, but the smallest in population within the large
Western Desert of Egypt. This Oasis is located in the central part of the Western Desert of Egypt approximately midway between Dakhla and Bahariya oases and covers an area of about $12000 \mathrm{~km}^{2}$.

Green, (1992) detected that, integration of Remote Sensing (RS) within the Geographic information system (GIS) database can decrease the cost, reduce 
the time and increase the detailed information gathered for soil survey. Particularly, the use of Digital Elevation Model (DEM) is important to derive landscape attributes that are utilized in land forms characterization, (Brough, 1986 and Dobos et al., 2000).

Digital Elevation Model (DEM) is a computerize model of the Earth's surface that can be stored and manipulated in a computer, (Brough, 1986). It provides greater functionalities than the qualitative and nominal characterization of topography. A DEM can be manipulated to provide many kinds of data that can assist the soil surveyor in mapping and giving a quantitative description of landforms and of soil variabilities.

Due to its geographical location and geological formation Farafra Oasis has more than $\mathbf{1 0 0}$ natural springs and wells spread out over the lands of the Farafra, many of which are natural. Most of these wells are used in irrigation of the cultivated land in the oasis.

The present study aims to produce the geomorphic map of El- Farafra Oasis and to identify the physical, chemical and mineralogical composition of the sand and clay fractions in order to evaluate the genesis and degree of homogeneity for soils in the study area.

\section{MATERIALS AND METHODS}

1-General description of the studied area

\section{a) Location}

The studied area is located between longitudes $27^{\circ} 49^{\prime} 19.18^{\prime \prime}$ to $28^{\circ} 04^{\prime} 41.28^{\prime \prime}$ E. and latitudes $26^{\circ} 46^{\prime} 10.98^{\prime \prime}$ to $27^{\circ} 22^{\prime}$ 38.75 " N.; it represents an area of about 372062 Feddans, (Figure, 1).

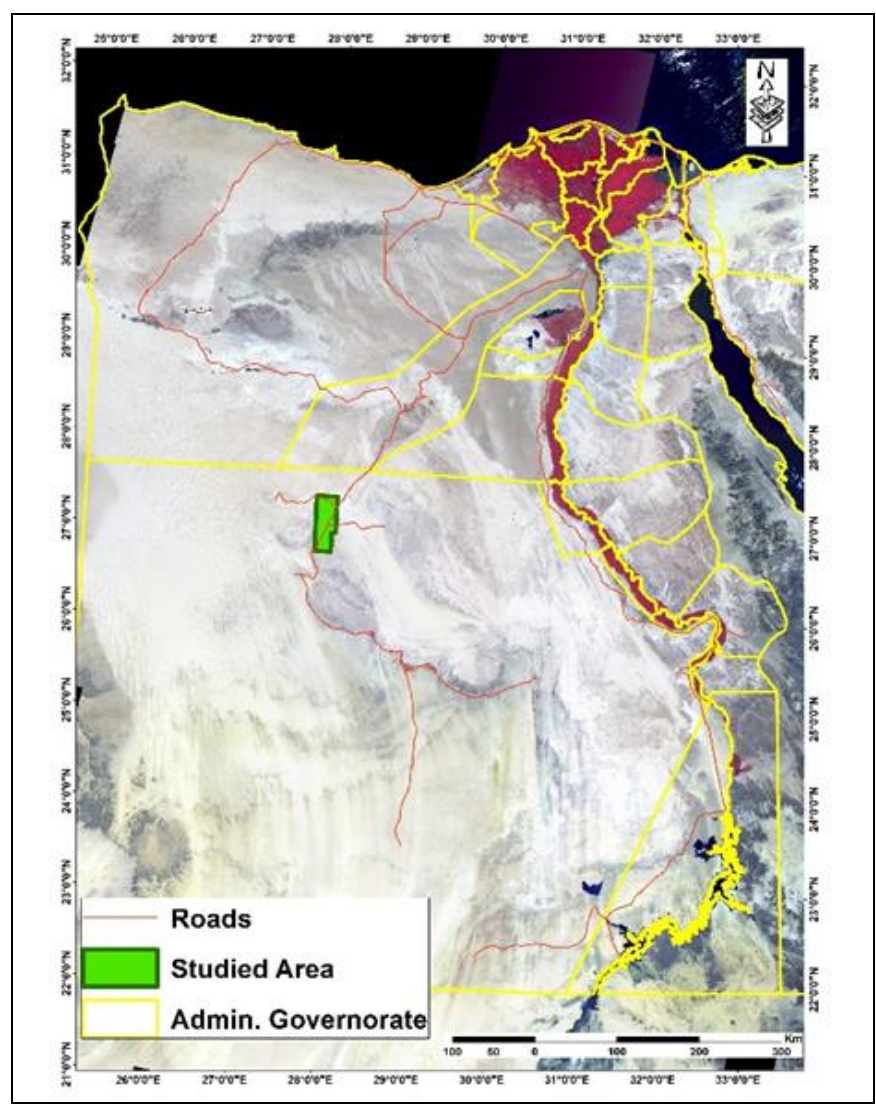

Figure (1): Location map of the studied area. 


\section{b) Geology:}

Sanad, (1972) stated that, the rock unit belonging to lower-middle Eocene in Farafra limestone. Conformably, it overlies the Esna shale and spreads overall the escarpments surrounding the depression. The rock unit belonging to the lower Eocene is Esna shale and covers the scarp- slopes surround the whole depression of old Farafra. It overlies the Farafra limestone and is under lowed by the Bishwa formation.

According to the geological map (EGSA, 1988) Khoman Chalkis the main formation which represents an area of about 188956 Feddans $(50.79 \%$ of the total studied area), followed by Dakhla formation (23.9\% of the total studied area) as shown in Figure (2) and Table (1).

\section{c) Digital Elevation Model (DEM)}

The contour lines and spot heights of geo-statistical analysis are used to produce the DEM of the studied area (Figure, 3) through interpolation Kriging inverse distance method, which used the semi-variogram parameters (Stein, 1998). The height areas located in the northwest side with elevation ranged from 279 to 350 meter above sea level. The low areas located in the eastern side with elevation between 47 and 83 meter above sea level.

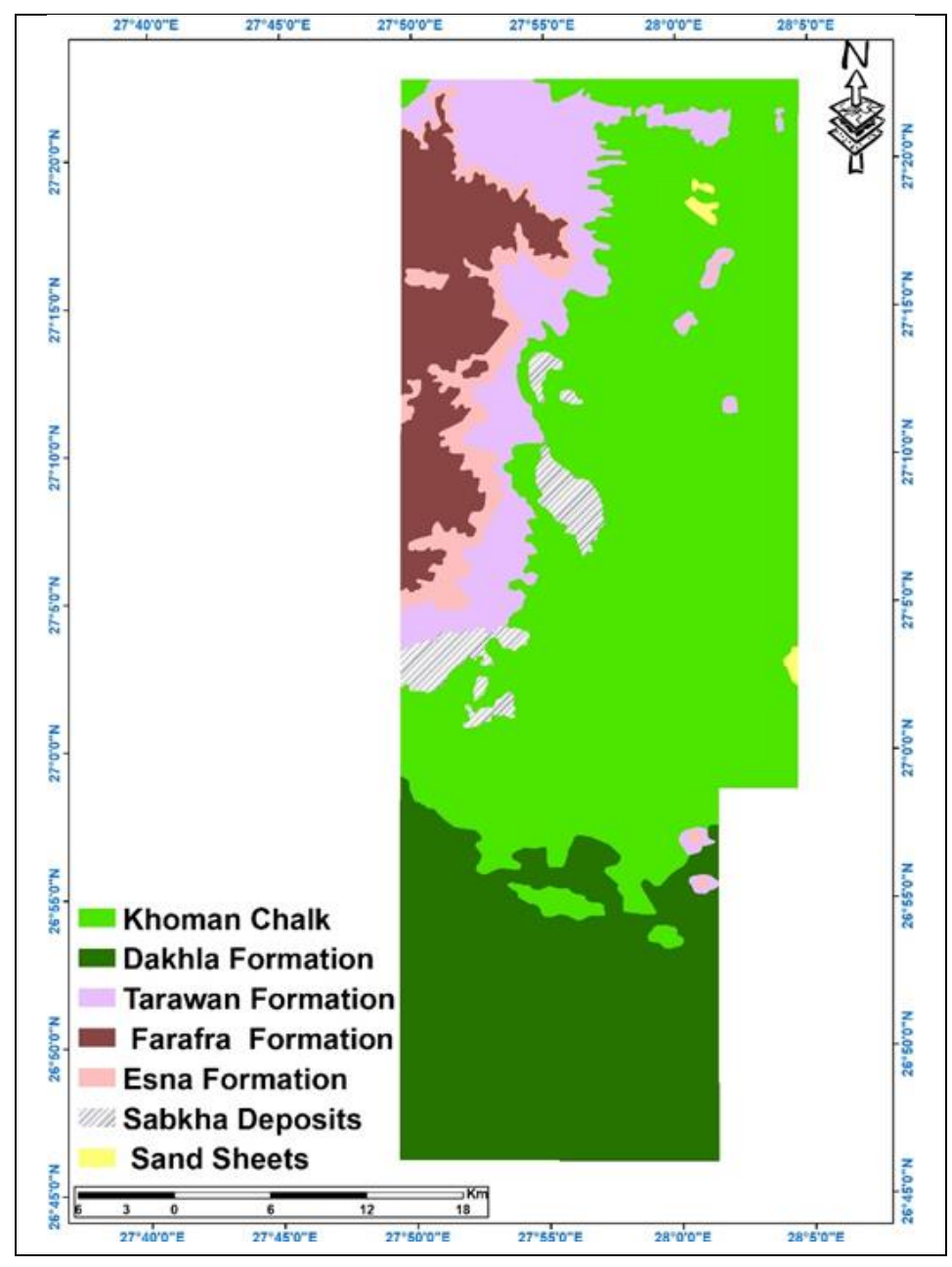

Figure (2): Geological map of the studied area (scale 1:500000) 
M. M. Soliman, et al.,

Table (1): Geological formations of the studied area (EGSA, 1988).

\begin{tabular}{|l|c|c|}
\hline \multicolumn{1}{|l|}{ Geological Formation } & Area (Fed.) & $\%$ \\
\hline Khoman Chalk & 188956 & 50.79 \\
\hline Dakhla Formation & 88917 & 23.90 \\
\hline Tarawan Formation & 38164 & 10.26 \\
\hline Sarafra Formation. & 30986 & 8.33 \\
\hline Sand Sha Deposits & 14573 & 3.92 \\
\hline Total & 9494 & 2.55 \\
\hline
\end{tabular}

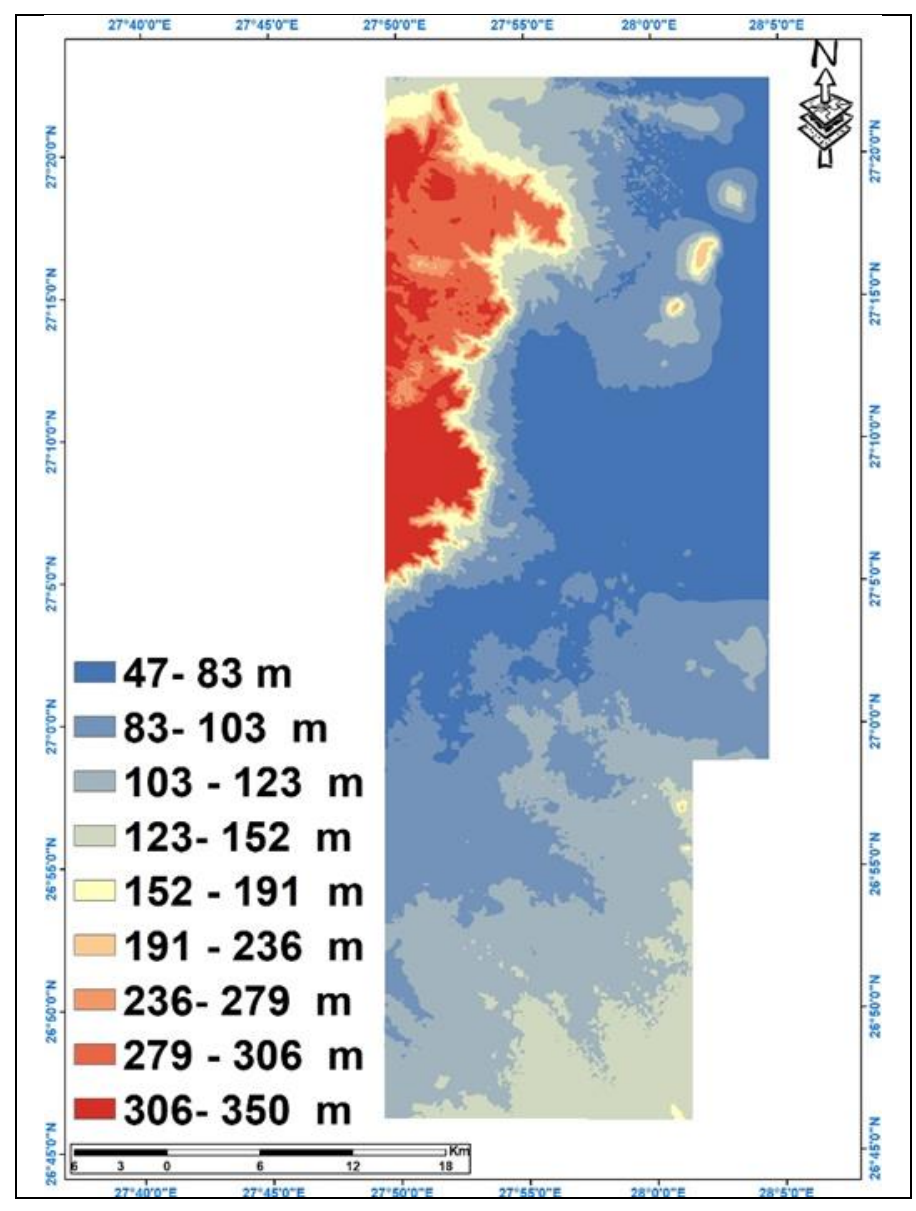

Figure (3): Digital Elevation Model (DEM) of the studied area. 


\section{d) Satellite data:}

Satellite image of the studied area derived from Sentinel 2 dated 20 May 2018 with spatial resolution of $10 \mathrm{~m}$ (Figure, 4) and spectral resolution of the bands 5,3 and 2 were used for delineating the geomorphic map (Zinck, 1988) of the studied area using the visual analysis, by aid of topographic maps, geology map and Digital Elevation Model (DEM). Spatial enhancement was done to produce an output image with enhanced edges that related to soil. The pixel values are not manipulated individually but in relation to their four neighbors. This modifies the value of each pixel on neighboring brightness values (Daels, 1986). Color enhancement was conducted to create new images from original to increase the amount of information that can be visually interpreted from the data.

The data and the output maps used the parameters for GIS displays from Egyptian Transverse Mercator projection (ETM) (Daels, 1986).

\section{Field Work:}

Eighty-two minipits were dug for checking the boundaries between geomorphic units and eighteen soil profiles were chosen to represent the different geomorphic units of the studied area. Soil representative samples from the different layers of the studied soil profiles were taken for laboratory analyses

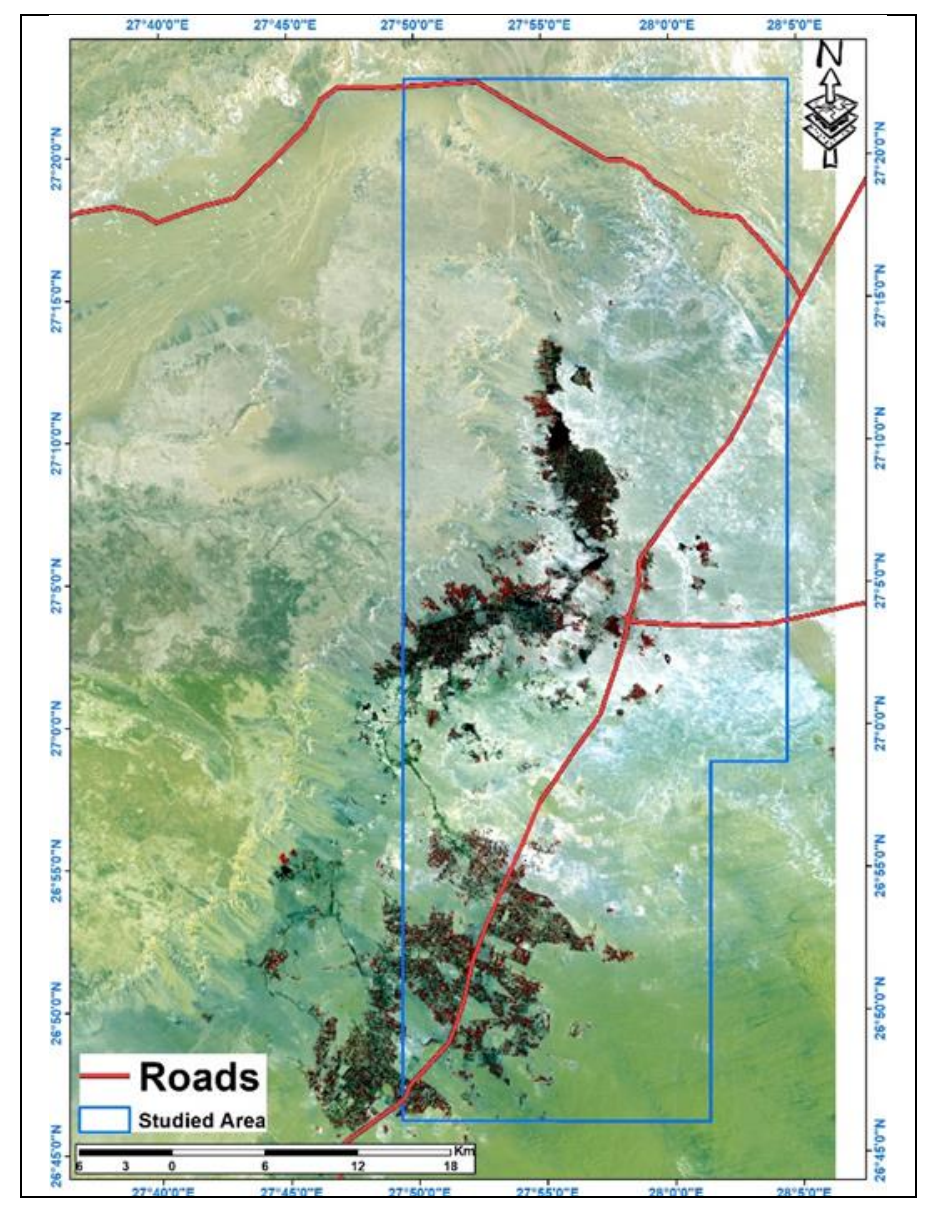

Figure (4): Satellite image of the studied area. 


\section{Laboratory Analyses:}

The collected disturbed soil samples were air dried, crushed and prepared for laboratory analyses, to determine some soil chemical and physical properties (Burt, 2004).

Particle size distribution was conducted using the pipette method. Calcium carbonate content was assessed using Collin's Calcimeter. Gypsum content was carried out by precipitation with acetone. Soil pH in the soil paste, salinity as electrical conductivity $\mathrm{EC}_{\mathrm{e}}$ in the soil paste extract, cation exchange capacity and exchangeable sodium percentage were carried out according to (Burt, 2004). The results are discussed as weighted profile mean for each geomorphic unit.

Mineralogical studies of the sand fraction were carried out as follows: after the ordinary treatment (Jackson, 1973); the sand fraction $(0.125-0.063 \mathrm{~mm})$ was separated from each sample by dry sieving methods. Separation of light and heavy minerals was carried out following the procedure described by Brewer, (1964). Mounting of the minerals was undertaken according to the method by Brewer, (1964); Systematic identification of the light and heavy minerals was done using the optical properties as given by Milner, (1962).

The mineralogical analysis of clay fraction $(<2 \mu)$ was carried out on ten representative soil samples to declare the clay minerals content of the different geomorphic units. Clay fraction $(<2 \mu)$ was separated by setting technique (Folk, 1980). The separated clay samples were slightly crushed and representative portion was disintegrated and dispersed by soaking in distilled water and stirring. The natural separated clay fraction was water precipitated into glass slides to produce preferred orientation of the clay minerals. Oriented slides were prepared and examined as follows: Mg-saturated air dried, Mg-saturated glycerol solvated, Ksaturated air dried and K-saturated after heating to $550{ }^{\circ} \mathrm{C}$ for four hours.

The X-ray diffraction analysis (XRD) was carried out using Scintag, Inc., U.S.A, $\mathrm{X} 1$, Advanced Diffraction system with $\mathrm{Cu}$ radiation and $\mathrm{Ni}$ filter. The XRD data were interpreted using ASTM cards together with data published by Brindly and Brown, (1980); schemes adopted by Jackson, (1973) and Dixon and Weed, (1977). Semiquantitative (relative proportions) of clay minerals identified were estimated by measuring the peak areas of the first order basal reflection (001) and calculating the percentages of frequency according to the methods outlined by (Weaver, 1961; Biscays, 1965; Gjems, 1967 and Venkatarathnam and Ryan, 1971).

\section{RESULTS AND DISCUSSION}

\section{Geomorphic units}

Visual interpretation of Sentinel 2 image was done on false color composite of bands 5, 3, 2 scale 1:50000 to produce a base map according to the difference in landscape from the field work activities. The integration between geology, Digital Elevation Model (DEM) and visual interpretation was carried out to produce a base map. This base map was used in the field to check, confirm, correct and modify the geomorphic unit boundaries, coupled with the results of the field work to produce final geomorphic map of the studied area (Figure, 5 and Table, 2).

There are two geomorphic units in the studied area namely Plateau (PL), and Depression (D). The plateau (pl) unit have about $9.74 \%$ of the total studied area with flat to almost flat topography. The Depression (D) unit have about $90.26 \%$ of the total studied area and consists of five sub units namely, chalky plain, peni plain, playa, sand sheets and dry wadis. 


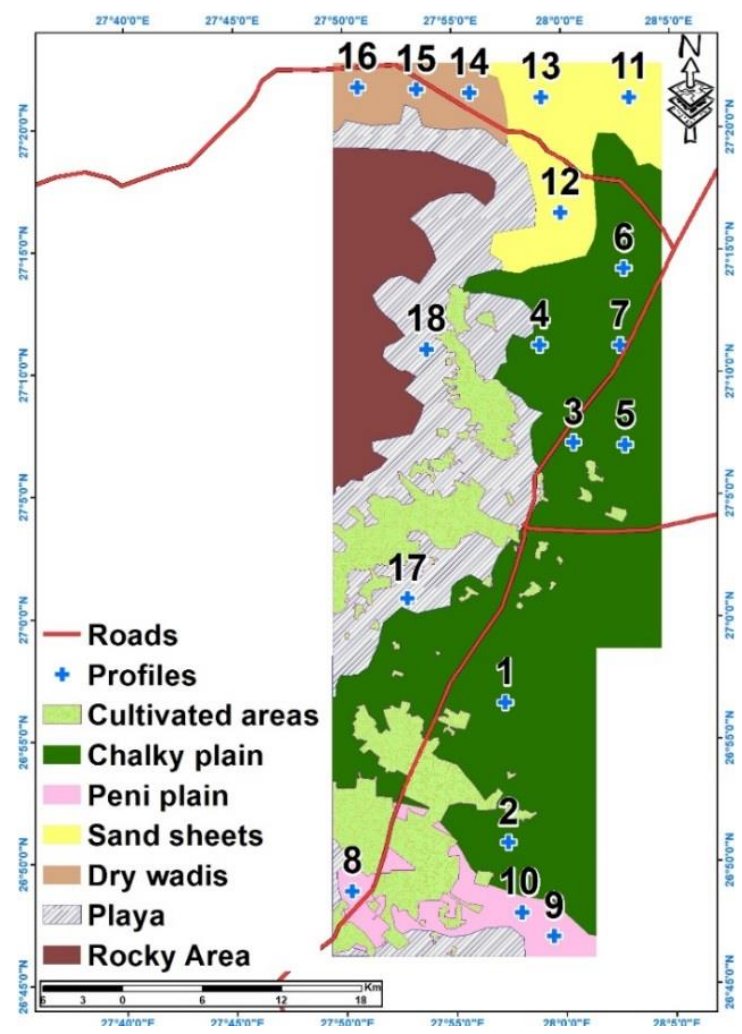

Figure (5): Geomorphic map and location of soil profiles.

Table (2): Geomorphic units of the studied area.

\begin{tabular}{|c|c|c|c|c|c|}
\hline$\underset{\text { unit }}{\text { Geomorphic }}$ & Relief & Lithology & $\begin{array}{l}\text { Sub units } \\
\text { landforms }\end{array}$ & $\begin{array}{l}\text { Area } \\
\text { (Fed.) }\end{array}$ & $\%$ \\
\hline Plateau (PL) & $\begin{array}{r}\text { Almost } \\
\text { flat } \\
\text { PL1 }\end{array}$ & $\begin{array}{c}\text { Limestone mixed } \\
\text { with } \\
\text { Shale (pl11) }\end{array}$ & $\begin{array}{c}\text { Summit (Rocky Area) } \\
\text { (pl111) }\end{array}$ & 36253 & 9.74 \\
\hline \multirow{6}{*}{$\begin{array}{l}\text { Depression } \\
\text { (D) }\end{array}$} & \multirow{3}{*}{$\begin{array}{c}\text { Almost } \\
\text { flat to } \\
\text { gently } \\
\text { undulat } \\
\text { ing } \\
\text { D1 }\end{array}$} & \multirow{3}{*}{$\begin{array}{l}\text { Limestone mixed } \\
\text { with shale and } \\
\text { sand stone (D11) }\end{array}$} & Chalky plain (D111) & 154918 & 41.64 \\
\hline & & & Peni plain (D112) & 15552 & 4.18 \\
\hline & & & Playa (D113) & 68904 & 18.52 \\
\hline & $\begin{array}{l}\text { Almost } \\
\text { flat D2 }\end{array}$ & Sandstone(D21) & Sand sheets (D211) & 30628 & 8.23 \\
\hline & $\begin{array}{l}\text { Undula } \\
\text { ting D3 }\end{array}$ & Limestone (D314) & Dry wadis (D311) & 14246 & 3.83 \\
\hline & $\begin{array}{l}\text { Almost } \\
\text { flat D4 }\end{array}$ & $\begin{array}{l}\text { Limestone mixed } \\
\text { with sand stone } \\
\text { (D41) }\end{array}$ & $\begin{array}{l}\text { Cultivated areas } \\
\text { (D411) }\end{array}$ & 51561 & 13.86 \\
\hline Total & & & & 372062 & 100.00 \\
\hline
\end{tabular}




\section{Soil properties of geomorphic sub} units:

\section{Chalky plain subunit:}

This subunit was represented by profiles 1, 2, 3, 4, 5, 6 and 7. Chalky plain is found in the depression floor as erosional remnants of the chalk. Data in Table (3) reveals that, texture classes of the representative soil profiles of this unit varied from loamy sand to sandy clay loam (as weighted mean). Calcium carbonate $\left(\mathrm{CaCO}_{3}\right)$ content varied were between 1.25 to $9.56 \%$ (as weighted mean). Organic matter (OM) was very low and ranged between from 0.09 to $0.89 \%$.

The soils are slightly alkaline where pH values varied from 7.21 to 7.79. ECe values varied from 1.16 to $22.02 \mathrm{dS} / \mathrm{m}$, indicating non-saline to highly saline soils. ESP ranged from 0.14 to $8.16 \%$, indicating no sodicity effect. Gypsum content ranges from 0.25 to $1.82 \%$. It may be concluded that, the parent material is limestone with almost physical weathering rather than chemical one and the leaching processes are weak thus, the fine fractions are very low.

Profile 5 was an exception with one layer $(0-25 \mathrm{~cm})$. Its data of $\mathrm{CaCO}_{3}, \mathrm{OM}, \mathrm{pH}$, EC, gypsum and ESP were $33.75 \%, 0.52 \%$, 7.73, $106 \mathrm{dS} / \mathrm{m}, 5.55 \%$ and $58.2 \%$, respectively.

\section{Peni plain subunit:}

This sub unit was represented by profiles 8, 9 and 10. Soil texture was varied between clay in profiles 8 and 9, and loamy sand in profile 10 (Table, 3). $\mathrm{CaCO}_{3}$ content ranged from 4.75 to $18.81 \%$ (as weighted mean). OM content is very low (between 0.13 to $0.54 \%$ ). Data in Table (3) revealed slightly alkaline soils as indicated from their $\mathrm{pH}$ values that ranged from 7.37 to 7.57. The soils are highly to very highly saline which having ECe values between 9.04 to $29.35 \mathrm{dS} / \mathrm{m}$. ESP ranges from 13.99 to $29.35 \%$ indicating sodicity effect. Gypsum content ranged from 0.64 to $5.02 \%$.

\section{Playa sub unit:}

This unit was represented by profiles 17 and 18. The playa deposits are composed of horizontal, alternating bands of soft, friable sand, clay and silt with frequent plant remains. Soil texture ranged between loamy sand to clay. $\mathrm{CaCO}_{3}$ content ranged from 7.78 to $9.85 \%$. OM content was very low (0.04 to $0.27 \%$ ). Data in Table (3) revealed slightly alkaline soils reaction with $\mathrm{pH}$ values ranged from 7.19 to 7.91. These soils were extremely saline as indicating from their ECe values that ranged from 42.42 to $59.58 \mathrm{dS} / \mathrm{m}$. ESP was between 31.85 and $38.39 \%$ indicating sodicity effect. Gypsum content is very low and ranged from 0.72 to $1.16 \%$.

\section{Sand sheets sub unit:}

This unit was represented by profiles 11, 12 and 13. Data in Table (3) revealed that, the soil texture varied from sand to sandy clay loam. $\mathrm{CaCO}_{3}$ content ranged from 3.13 to $37.08 \%$. OM is very low not exceeds $0.39 \%$. The $\mathrm{pH}$ values varied from $\mathbf{7 . 3 5}$ to $\mathbf{7 . 8}$ indicating slightly alkaline soil reaction. These soils are highly to extremely saline indicating from their ECe values that ranged between 20.16 and $63.83 \mathrm{dS} / \mathrm{m}$. The soils are sodic affected with ESP values varied from 12.69 to $30.48 \%$. Gypsum content is very low and ranged from 0.35 to $1.29 \%$.

\section{Dry wadis subunit:}

This unit was represented by profiles 14, 15 and 16. The Wadi is filled with alluvial materials developed through weathering of parent rock and transported by floodwater to the lowlands. Data in Table (3) showed that, the soil have sandy clay loam to clay texture. $\mathrm{CaCO}_{3}$ content ranged from 7.14 to $9.83 \%$. OM content is very low not exceeds $0.49 \%$. Soil $\mathrm{pH}$ ranged from 7.39 to 7.66 indicating slightly alkaline reaction. ECe varied 


\begin{tabular}{|c|c|c|c|c|c|c|c|c|c|c|c|c|c|}
\hline & & & 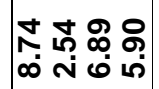 & 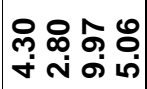 & 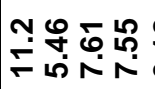 & 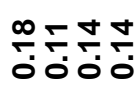 & พุ: & 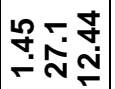 & 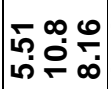 & Tَog & 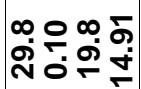 & 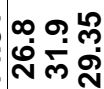 & \\
\hline & & & 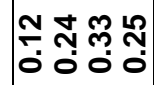 & 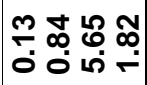 & 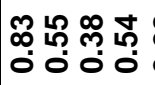 & 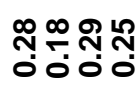 & 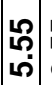 & 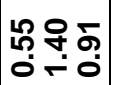 & సิำ: & 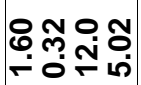 & 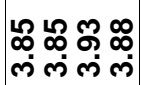 & 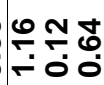 & \\
\hline & & & 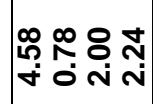 & 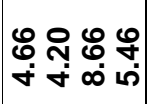 & 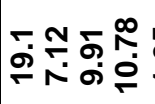 & & $\begin{array}{l}0 \\
\dot{0} \\
0\end{array}$ & 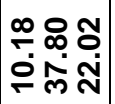 & 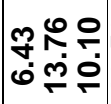 & $\mid$ & 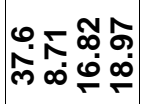 & 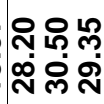 & \\
\hline & & & 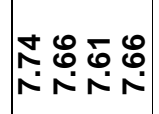 & 导 & $\mid$ & فำ & $\stackrel{\substack{n \\
r}}{2}$ & 家奇 & कㅇำ & 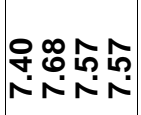 & 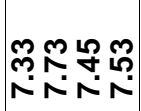 & ㄲํำ & \\
\hline & & & $\left|\begin{array}{cccc}\infty & 0 & 0 \\
\infty & \ddots & 0 & 9 \\
0 & 0 & - & 0 \\
0\end{array}\right|$ & 농 & 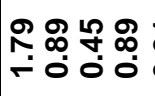 & 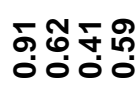 & ஸิ & 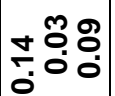 & 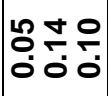 & 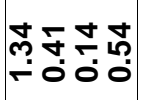 & 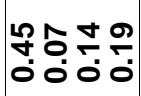 & 풍 & $\frac{\mathbb{Z}}{\pi}$ \\
\hline & & & শุ丷 & 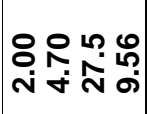 & 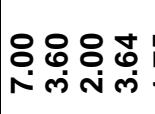 & مִ & $\mid \begin{array}{c}\mathfrak{n} \\
\mathfrak{m} \\
\tilde{m}\end{array}$ & 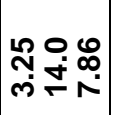 & ס्र & 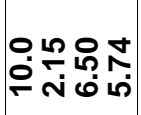 & 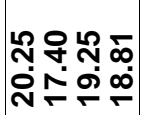 & 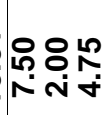 & 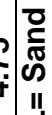 \\
\hline & & & ల్ల ల్ల ల్ల & のららら & 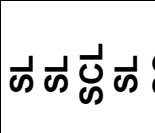 & రூఝळの & u্s & の๘の & Ј๘ & OOOC & 000 & תפ & $\left.\right|_{\infty} ^{\infty}$ \\
\hline & ळ & $\frac{\pi}{0}$ & 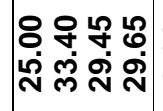 & 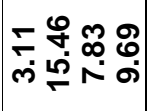 & 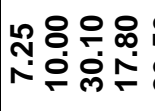 & 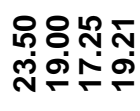 & 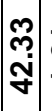 & 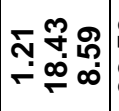 & 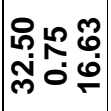 & 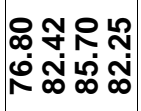 & 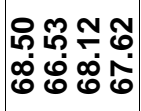 & 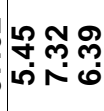 & on \\
\hline & 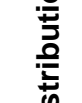 & 志 & 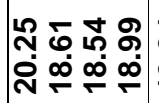 & 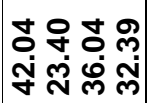 & 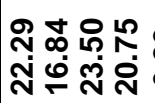 & 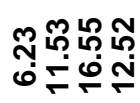 & ని & רิ & 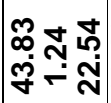 & 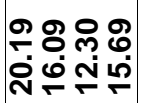 & 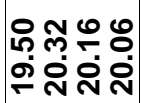 & 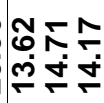 & $\hat{\varepsilon}$ \\
\hline & $\begin{array}{l}\frac{\mathbb{N}}{N} \\
\frac{\Xi}{0}\end{array}$ & Oִ & 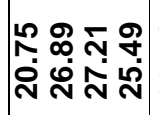 & 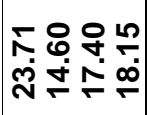 & 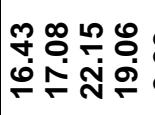 & 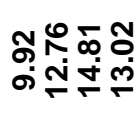 & 文 & 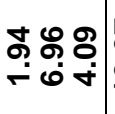 & ڤัن & 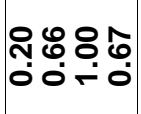 & 员 & 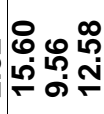 & 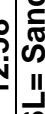 \\
\hline & $\frac{1}{0}$ & $\ddot{0}$ & 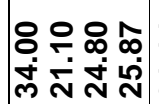 & 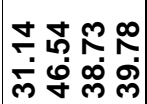 & 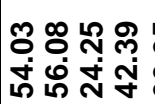 & 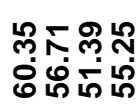 & 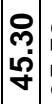 & 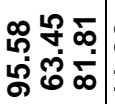 & 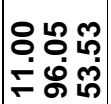 & 我 & 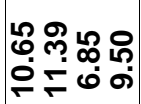 & 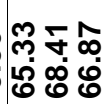 & \\
\hline & & & 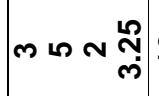 & 으 $\infty \cong \underset{0}{0}$ & O & ๓ 농 & $\mp$ & 으 ᄂ & mas & 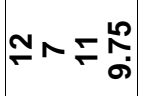 & 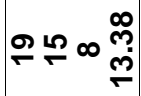 & $+\infty \frac{8}{6}$ & (ill \\
\hline & & & 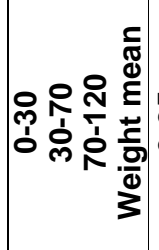 & 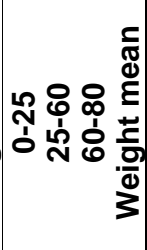 & 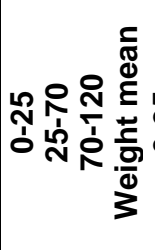 & 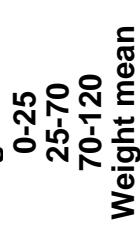 & & 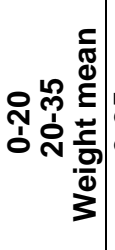 & مُ & 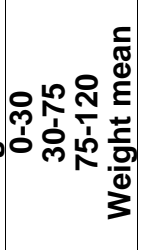 & 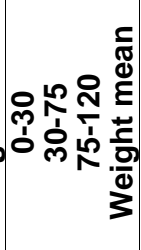 & 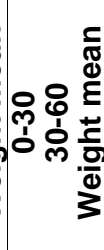 & $=$ \\
\hline & & & - & N & $m$ & $\theta$ & in & 0 & $\wedge$ & $\infty$ & 0 & 우 & \\
\hline & $\begin{array}{l}\text { sl!u } \\
\text { ग!ydı }\end{array}$ & & & & u!̣eld אYy & 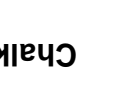 & & & & & 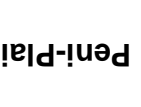 & & U \\
\hline
\end{tabular}


M. M. Soliman, et al.,

\begin{tabular}{|c|c|c|c|c|c|c|c|c|c|}
\hline \multicolumn{2}{|c|}{ 号 } & 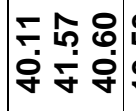 & 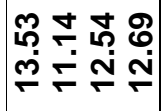 & गे N & ஸे & 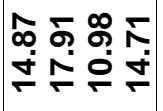 & 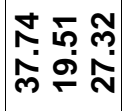 & 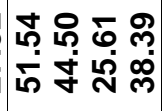 & 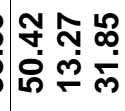 \\
\hline \multicolumn{2}{|c|}{ 엉 } & 象 & 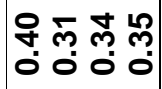 & | & ָָ & 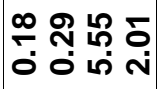 & L̊ำ & 贷 & ูำ \\
\hline \multicolumn{2}{|c|}{ 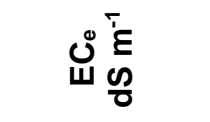 } & 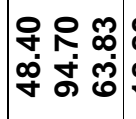 & 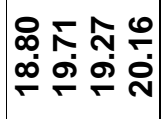 & 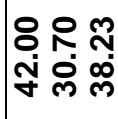 & ণ্ণ & 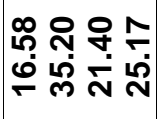 & 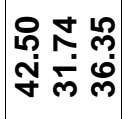 & 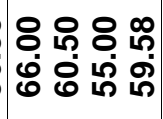 & กั \\
\hline \multicolumn{2}{|c|}{ 工 동 } & 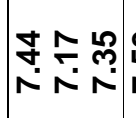 & 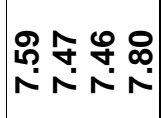 & 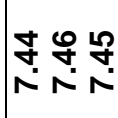 & 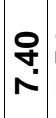 & 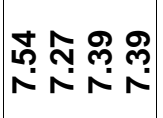 & 员芯 & ํㅜㄴ & רס \\
\hline \multicolumn{2}{|c|}{ ¿o } & 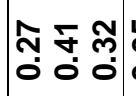 & 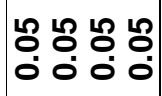 & 웜 & L̊ & 음 №음 & 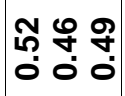 & 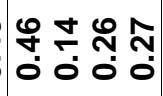 & 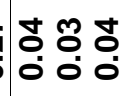 \\
\hline \multicolumn{2}{|c|}{ ర్ల్ర } & 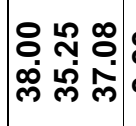 & 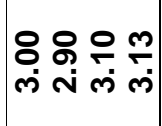 & $\begin{array}{l}10 \\
\infty \\
\infty \\
0\end{array}$ & $\begin{array}{l}8 \\
6 \\
\sigma\end{array}$ & 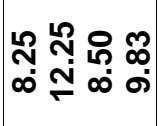 & 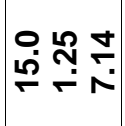 & 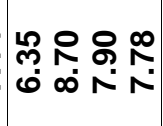 & 突 \\
\hline \multicolumn{2}{|c|}{ 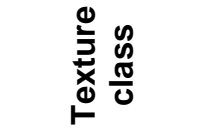 } & ○ ల్ల & $\cos \cos \theta$ & ๘あ山 & 0 & 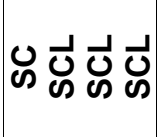 & ப ப & のらの & 000 \\
\hline \multirow{4}{*}{ 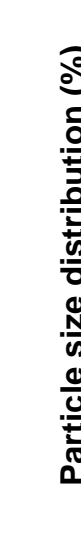 } & $\frac{\pi}{0}$ & 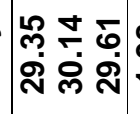 & 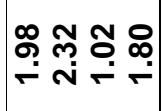 & 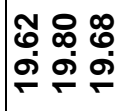 & ঙ̊ & 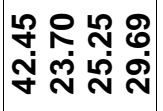 & 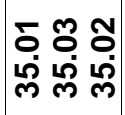 & 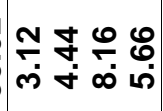 & 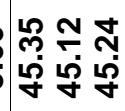 \\
\hline & $\frac{5}{\omega}$ & 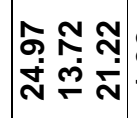 & 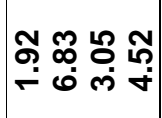 & | & $\begin{array}{l}\hat{0} \\
\dot{\omega} \\
\text {. }\end{array}$ & 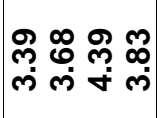 & 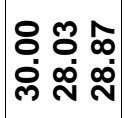 & 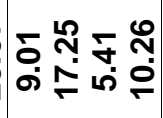 & 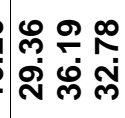 \\
\hline & $\stackrel{0}{\mathscr{0}}$ & 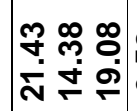 & 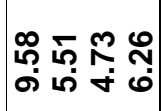 & 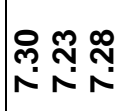 & $\begin{array}{l}\stackrel{0}{0} \\
\dot{\sim} \\
\end{array}$ & 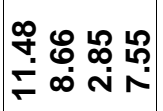 & 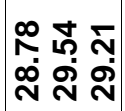 & 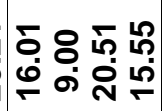 & 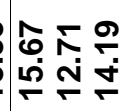 \\
\hline & on & 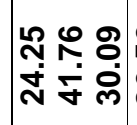 & 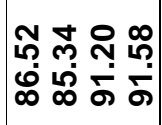 & 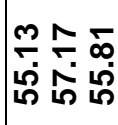 & $\underset{\infty}{\infty}$ & 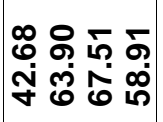 & 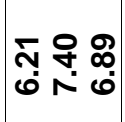 & 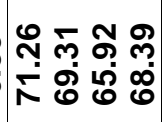 & 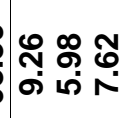 \\
\hline \multicolumn{2}{|c|}{ 悹 。 } & N 우 $\stackrel{\hat{\sigma}}{\dot{q}}$ & N L L ᄂ & m $\infty$ ஸे & N & 우 는 돔 & 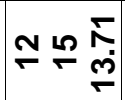 & $\ln \sim \sim \frac{n}{N}$ & N \\
\hline \multicolumn{2}{|c|}{ 产 } & |cc| & 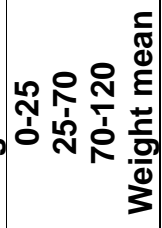 & 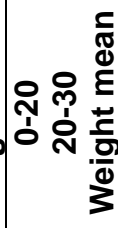 & ô & 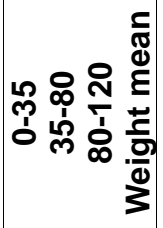 & 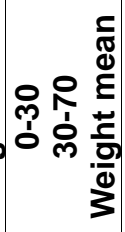 & 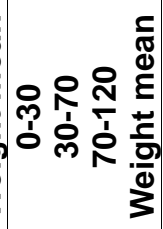 & 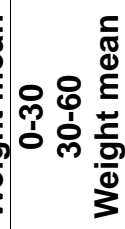 \\
\hline \multicolumn{2}{|c|}{ ஸ் 웅 } & $F$ & $\stackrel{\Upsilon}{\Upsilon}$ & $\stackrel{m}{\longrightarrow}$ & I & $\stackrel{20}{\leftarrow}$ & $\stackrel{0}{\circ}$ & $\mp$ & $\stackrel{\infty}{\infty}$ \\
\hline \multicolumn{2}{|c|}{$\begin{array}{c}\text { s!!unqns } \\
\text { ग!५dıomoəə }\end{array}$} & \multicolumn{3}{|c|}{ toous pues } & \multicolumn{3}{|c|}{ s!pem Kua } & \multicolumn{2}{|c|}{ exeld } \\
\hline
\end{tabular}


between $19.20 \mathrm{dS} / \mathrm{m}$ (highly saline) to $36.35 \mathrm{dS} / \mathrm{m}$ (extremely saline). ESP values ranged from 14.71 to $27.32 \%$ (sodicity effect). Gypsum content is low and varied from 0.28 to $2.01 \%$.

\section{Mineralogical Analyses}

\section{Mineralogy of the clay fraction}

Clay fraction content of the soil is quite important for agriculture land use. It constitutes the most active part in soil as well as controls the majority of the physical and chemical properties. Therefore, studying the mineralogical composition of the clay fraction is very important to recognize the nature and types of clay mineralogy that strongly affects the soil fertility and development.

\section{Chalky plain:}

The mineralogical composition of the clay fraction separated from the soils of chalky plain (profiles 1 and 3 ) is dominated by Kaolinite with less amounts of smectite. Illite mineral is detected in few amounts in profile 1 (Table, 4). While it absented in profile 3 . Interstrstratified minerals are found in few amounts in the $70-120 \mathrm{~cm}$ depth of profile 3.

\section{Peni plain:}

X-ray diffraction pattern of the clay fraction separated from the peni plain soils (profile 8 ) are characterized by an abundance amount of kaolinite followed by smectite. Interstrstratified minerals are detected in trace amounts.

\section{Playa:}

The mineralogy of the clay fraction separated from the (30-70) layer of profile 17 that representing this unit was dominated by kaolinite mineral. Smectite and Interstratified minerals are detected in trace amount. Chlorite mineral is detected in few amounts (Table, 4).

Table (4): Semi- quantitative estimation of the clay fraction $(<0.002 \mathrm{~mm})$ separated from some layers of the studied soil profiles.

\begin{tabular}{|c|c|c|c|c|c|c|c|c|c|}
\hline \multirow{2}{*}{ 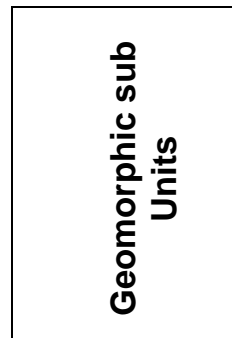 } & \multirow[b]{2}{*}{$\begin{array}{c}\text { Profile } \\
\text { No. }\end{array}$} & \multirow[b]{2}{*}{$\begin{array}{c}\text { Depth } \\
\text { (cm.) }\end{array}$} & \multicolumn{7}{|c|}{$\begin{array}{c}\text { Clay } \\
\text { minerals }\end{array}$} \\
\hline & & & 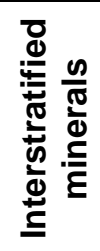 & 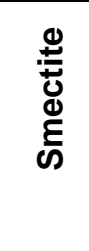 & 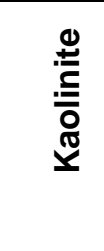 & 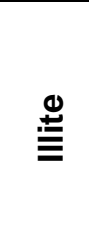 & 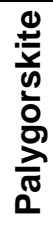 & 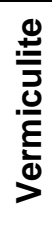 & $\begin{array}{l}\stackrel{0}{\frac{\pi}{2}} \\
\frac{0}{\frac{0}{\delta}}\end{array}$ \\
\hline \multirow{4}{*}{$\begin{array}{l}\text { Chalky- } \\
\text { Plain }\end{array}$} & \multirow[b]{2}{*}{1} & $30-70$ & -- & -- & Dom & Few & -- & -- & -- \\
\hline & & $70-120$ & -- & Few & Dom & Few & -- & -- & -- \\
\hline & \multirow[b]{2}{*}{3} & $25-70$ & -- & Tra & Few & -- & -- & -- & -- \\
\hline & & $70-120$ & Few & - & Mod & - & -- & -- & -- \\
\hline \multirow{2}{*}{ Peni-plain } & \multirow[b]{2}{*}{8} & $30-75$ & -- & Tra & Few & -- & -- & -- & -- \\
\hline & & $75-120$ & Tra & Few & Mod & -- & -- & -- & -- \\
\hline Sand sheets & 11 & $0-20$ & Few & Tra & Few & Few & -- & -- & Few \\
\hline \multirow{2}{*}{ Dry Wadis } & \multirow[b]{2}{*}{15} & $35-80$ & Tra & Tra & Few & - & -- & -- & Few \\
\hline & & $80-120$ & Few & Few & Dom & -- & -- & -- & -- \\
\hline Playa & 17 & $30-70$ & Tra & Tra & Few & -- & -- & -- & -- \\
\hline
\end{tabular}

Notes: --: Absent Tra.: trace <5\%; Few: 5-15\% Mod.: Moderate (15-25\%) Com.: Common (25-40\%); Dom.: Dominate $>40 \%$. 


\section{Sand Sheets:}

The study of clay minerals for the soils of Sand sheets unit which are represented by the layer of (0-20) in profile 11. Data in Table (4) showed that, kaolinite pre dominated the clay minerals suite in these layers. Interstratified minerals and chlorite were found in a few amounts. Smectite minerals are detected in trace amount.

\section{Dry wadis:}

Data in Table (4) revealed that, the clay fraction of dry wadis soils is dominated by kaolinite (about $80 \%$ ) followed by interstratified minerals. Smectite minerals are found in trace amount in the subsurface layer and increased to few amounts in the deepest layer. Chlorite mineral is detected in a few amounts in the subsurface layer and disappeared in the deepest layer.
In conclusion it is well known that the clay minerals could be present as a result of inheritance from parent material by alteration degradation of primary minerals synthesizing and addition. For the present study, the chemical decay is somewhat limited due to the prevalence of aridity, while the mechanical weathering contributes to a great extent. The Variation in the relative content of present clay minerals may be attributed mainly to sedimentation regime varieties and / or to the nature of the source rocks.

\section{Mineralogy of the sand fraction}

Tables (5 and 6 ) show the distribution of light and heavy minerals in the sand fraction of some soil profiles in the studied area as well as their distribution throughout the entire depth of each profile.

Table (5): Frequency distribution of the light minerals in the sand fraction (0.125-0.063 $\mathrm{mm}$.) in the studied soil profiles.

\begin{tabular}{|c|c|c|c|c|c|c|c|}
\hline \multirow[b]{2}{*}{$\begin{array}{l}\text { Geomorphic } \\
\text { sub-units }\end{array}$} & \multirow[b]{2}{*}{$\begin{array}{l}\text { Profile } \\
\text { No. }\end{array}$} & \multirow[b]{2}{*}{ Depth (cm.) } & \multirow[b]{2}{*}{$\begin{array}{c}\text { Quartz } \\
(\%)\end{array}$} & \multicolumn{3}{|c|}{ Feldspars (\%) } & \multirow[b]{2}{*}{$\begin{array}{c}\text { Total } \\
(\%)\end{array}$} \\
\hline & & & & 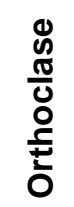 & $\begin{array}{l}\text { 刃 } \\
\frac{\pi}{0} \\
\frac{0}{\sigma} \\
\frac{\pi}{\alpha}\end{array}$ & 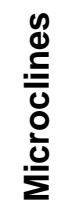 & \\
\hline \multirow{2}{*}{ Chalky Plain } & 1 & $\begin{array}{c}30-70 \\
70-120\end{array}$ & $\begin{array}{l}97.3 \\
95.6\end{array}$ & $\begin{array}{l}1.4 \\
2.6\end{array}$ & $\begin{array}{l}0.8 \\
1.3\end{array}$ & $\begin{array}{l}0.5 \\
0.5\end{array}$ & $\begin{array}{l}2.7 \\
4.4\end{array}$ \\
\hline & 3 & $\begin{array}{c}25-70 \\
70-120\end{array}$ & $\begin{array}{l}96.7 \\
96.2\end{array}$ & $\begin{array}{l}1.7 \\
2.1\end{array}$ & $\begin{array}{l}1.4 \\
1.5\end{array}$ & $\begin{array}{l}0.2 \\
0.2\end{array}$ & $\begin{array}{l}3.3 \\
3.8\end{array}$ \\
\hline Peni-plain & 8 & $\begin{array}{c}30-75 \\
75-120\end{array}$ & $\begin{array}{l}95.1 \\
97.6\end{array}$ & $\begin{array}{l}2.8 \\
1.3\end{array}$ & $\begin{array}{l}1.6 \\
0.7\end{array}$ & $\begin{array}{l}0.5 \\
0.4\end{array}$ & $\begin{array}{l}4.9 \\
2.4\end{array}$ \\
\hline Sand sheets & 11 & $\begin{array}{c}0-20 \\
20-30\end{array}$ & $\begin{array}{l}98.6 \\
98.2\end{array}$ & $\begin{array}{l}0.6 \\
1.1\end{array}$ & $\begin{array}{l}0.4 \\
0.5\end{array}$ & $\begin{array}{l}0.4 \\
0.2\end{array}$ & $\begin{array}{l}1.4 \\
1.8\end{array}$ \\
\hline Dry Wadis & 15 & $\begin{array}{c}35-80 \\
80-120\end{array}$ & $\begin{array}{l}97.5 \\
98.7\end{array}$ & $\begin{array}{l}1.2 \\
0.7\end{array}$ & $\begin{array}{l}0.7 \\
0.3\end{array}$ & $\begin{array}{l}0.6 \\
0.3\end{array}$ & $\begin{array}{l}2.5 \\
1.3\end{array}$ \\
\hline Playa & 17 & $\begin{array}{c}30-70 \\
70-120\end{array}$ & $\begin{array}{l}98.5 \\
97.8\end{array}$ & $\begin{array}{l}0.7 \\
1.1\end{array}$ & $\begin{array}{l}0.5 \\
0.8\end{array}$ & $\begin{array}{l}0.3 \\
0.3\end{array}$ & $\begin{array}{l}1.5 \\
2.2\end{array}$ \\
\hline
\end{tabular}




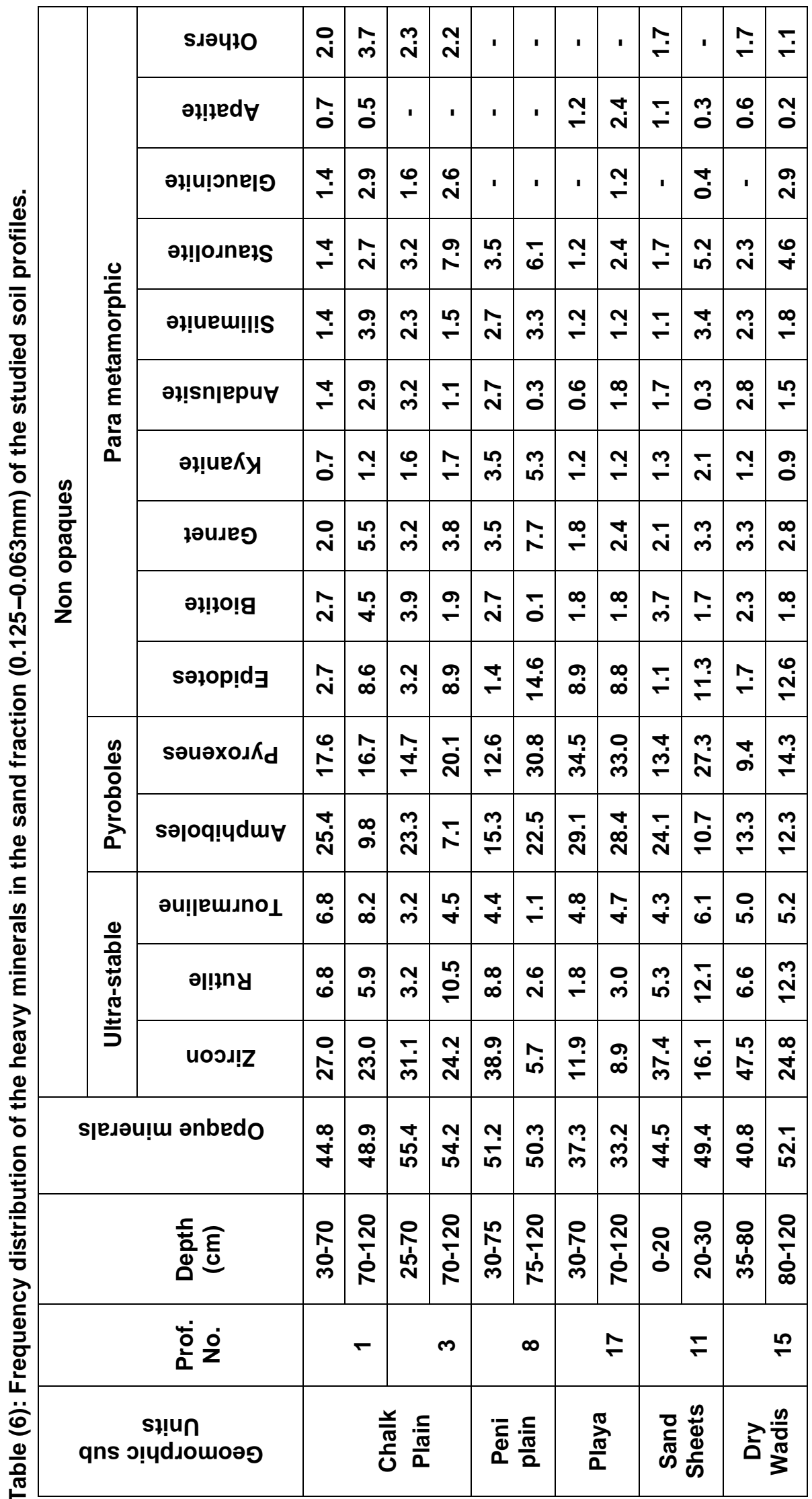


1- Light minerals (Sp. Gr $<2.85$ $\mathrm{g} / \mathrm{cm} 3$ ): They are composed almost entirely from quartz that constituted about $95 \%$. Other light minerals are mainly orthoclase, plagioclase and microcline. The dominancy of quartz over other members of the light minerals is mostly related to its resistance to weathering and disintegration during the multi cyclic processes of sedimentation. On the other hand, the presence of feldspars could be taken as indication for the weathering prevailed during the soil formation that was not so drastic to cause a complete decay of inerals susceptible to weathering.

2- Heavy minerals (Sp. Gr > 2.85 $\mathrm{g} / \mathrm{cm} 3$ ): The heavy minerals could be used as a tool to evaluate the source rocks, soil profile uniformity, and the state of mineral weathering. The frequency distribution of the heavy minerals (opaques and non-opaques) could be discussed in the same order as given in Table (6).

a) Opaque minerals:

Opaque's are mostly composed of iron ores such as hematite, ilmenite, limonite and pyrite. The opaque minerals constituted the greater part of the heavy minerals in all samples.

The frequency of opaque minerals seems to be dependent on the soil geographic distribution, weathering conditions and soil texture. The maximum value was detected in the soils of chalky plain $(55.4 \%)$, while the minimum one was recorded in the playa soils $(33.2 \%)$.

\section{b) Non-opaque minerals:}

The non-opaque minerals are those formed from the rocks containing them. The essential minerals constitute the major portion of rock and / or necessary to give its character as a whole. These minerals are prevailed as ferro - silicatepyroboles and epidote, while their accessory minerals are zircon, rutile ... etc.

i) Pyroboles:

a- Pyroxenes: Pyroxenes varied widely from $9.4 \%$ in the subsurface layer of profile 15 (Dry wadis) to $34.5 \%$ in the subsurface layer of profile 17 (playa). The variation of pyroxenes in the studied soils may be rendered to the nature of parent material, its discontinuity as well as sedimentation regimes and environments.

b-Amphiboles: The content of amphiboles ranged from 7.1 to $29.1 \%$. Their-lowest content were recorded in profile 3 (chalky plain), while the highest ones characterized the deepest layer of profile 17 (playa).

In conclusion, the abundance of amphiboles and pyroxenes in El- Farafra Oasis could be as indication for recent and poorly developed sediments that were derived mainly from western plateau rocks (metamorphic).

\section{ii) Ultra-stable minerals:}

a- Zircon: This mineral was the abundant ultra-stable mineral in most of the studied profiles. The highest value was recorded in the $35-80 \mathrm{~cm}$ layer of profile 15 (Dry wadis), while the lowest one was found in the deepest layer of profile8 (peni plain). The vertical distribution of zircon indicated no consistent pattern. This indicated the discontinuity of zircon contents with depth. Also, this reflects the multiorigin of parent material or its multidepositional pattern.

b- Rutile: It is considered as second abundance ultra-stable mineral in most of the studied soil profiles. Rutile has a frequency varied between 1.8 and $12.3 \%$, with no regular distribution 
pattern with soil profile depths. The apparent discontinuity in the mineral distribution could be explained multi origin of the studied soils that were derived from multi parent materials and different depositional regime.

c- Tourmaline: Data in Table (6) revealed that, tourmaline content in the studied area ranged from 1.1 to $8.2 \%$. The lowest value was detected in profile 8 (peni plain). While; the highest content was found in profile 1 (chalky plain). The mineral had an irregular distribution pattern the soil profiles depths.

In conclusion, the occurrence of ultrastable minerals in the heavy minerals means either (1), the minerals are being reworked from older sediments (Folk, 1968), or sedimentary rocks e.g. the Miocene sandstone of the western hills and / or (2) chemical attach has occurred. The active physical and inactive chemical weathering processes on multi - sources parent materials led to the prevalence of immature soil pedons in El- Farafra Oasis. This is indicated by the irregular distribution of the ultra-stable minerals in the soils of different geomorphic units and also by the irregular vertical distributions of such minerals.

iii) Para metamorphic minerals:

a- Garnet: Its highest values was found in profile 8 (peni plain), and the lowest content was in $\mathbf{3 0 - 7 0} \mathrm{cm}$ layer of profile (playa).

b- Staurolite: documented in all examined samples with variable percentages ranging from $1.2 \%$ in the subsurface layer of profile 17 (playa sediments) to $7.9 \%$ in the deepest layer of profile (chalky plain soils).

c- Kyanite: ranged from $0.7 \%$ in subsurface layer of profile 1 (Chalky plain), to $5.3 \%$ in the deepest layer of profile 8 (peni plain soils).

d- Silimanite: is the least abundant mineral among the para metamorphic minerals constitutes $1.1 \%$ to $3.9 \%$. The highest value was recorded in the subsurface layer of profile 1 (chalky plain), while the lowest value was found in the surface layer of profile 11 (sand sheets).

e- Biotite: constituted $0.1 \%$ to $4.5 \%$. The lowest value was detected in the deepest layer of profile 8 (peni plain soils). While the highest content was detected in the 70-120 cm layer of profile 1 (chalky plain soils). From the pedological view point, the presence of biotits as potassium- bearing mineral in the studied area is very important for clay mineral formation by the geochemical weathering processes.

f- Epidote: detected in all the studied samples with frequency ranging from 1.1 to $14.6 \%$. The relatively low amounts of epidote were recorded in the surface layer of profile 11 (sand sheets). The highest content was recorded in the deepest layer of profile 8 (peni plain soils).

g- Andolusite: ranged from 0.3 to $3.2 \%$ of the non- opaque minerals. The lowest value was recorded in the deepest layer of profile No.8 (peni plain). The highest value was found in the subsurface layer of profile 3 (chalky plain soils).

h- Glauconite: ranged from 0.4 to $2.9 \%$. It detected in minute amounts or sometimes absent in profile 3 and some layers of profiles 17,11 and15.

i- Apatite: recorded in a maximum frequency representing $2.4 \%$ of the non-opaque minerals in the deepest layer of profile 17 (Playa), The lowest 
content was recorded in the deepest layer of profile 15 (Dry wadis). It absents in chalky plain soils (profile 3 ) and peni plain (profile 8).

\section{Uniformity of soil materials}

Pyroboles, zircon, tourmaline and rutile minerals are used to evaluate the weathering ratio and uniformity of studied soils parent materials. Pyroboles considered as an assessable mineral to weathering. Zircon, tourmaline and rutile are considered as resistant minerals (Brewer, 1964). So the presence of assessable minerals in high percentage can be taken as an indication to the immature materials of the recent deposits. The mineral assemblage (Table, 6 ) as well as the ratios of $Z / T, Z / R, Z / T^{\star} R,(P+A) / Z+T$ and $B / Z+T$ are calculated for the studied profiles in order to evaluate their uniformity. Data in Table (7) reveal that, the studied soils of geomorphic units in EI- Farafra Oasis are formed from multiorigin and / or multi depositional regimes. This indicated by the irregular trend of both uniformity and weathering ratios with depth. On the other hand, weathering ratios indicated that, the studied soils are considered young (immature) from the pedological point of view.

Table (7): Weathering and uniformity ratios of the studied soil profiles.

\begin{tabular}{|c|c|c|c|c|c|c|c|}
\hline \multirow{2}{*}{$\begin{array}{l}\text { Geomorphic } \\
\text { subunits }\end{array}$} & \multirow{2}{*}{$\begin{array}{c}\text { Profile } \\
\text { No. }\end{array}$} & \multirow{2}{*}{$\begin{array}{l}\text { Depth } \\
\text { (cm.) }\end{array}$} & \multicolumn{3}{|c|}{$\begin{array}{l}\text { Uniformity } \\
\text { ratios }\end{array}$} & \multicolumn{2}{|c|}{$\begin{array}{l}\text { Weathering } \\
\text { ratios }\end{array}$} \\
\hline & & & Z/T & Z/R & $Z /(R+T)$ & $\begin{array}{c}W r 1 \\
(P+A) / Z+T\end{array}$ & $\begin{array}{c}\text { Wr2 } \\
(B) / Z+T\end{array}$ \\
\hline \multirow{4}{*}{ Chalky Plain } & \multirow{2}{*}{1} & $30-70$ & 4.0 & 4.0 & 2.0 & 1.3 & 0.05 \\
\hline & & $70-120$ & 2.8 & 3.9 & 1.6 & 0.8 & 0.14 \\
\hline & \multirow{2}{*}{3} & $25-70$ & 9.7 & 9.7 & 4.9 & 1.1 & 0.10 \\
\hline & & $70-120$ & 5.4 & 2.3 & 1.6 & 0.5 & 0.10 \\
\hline \multirow{2}{*}{ Peni Plain } & \multirow{2}{*}{8} & $30-75$ & 8.8 & 4.4 & 2.9 & 0.6 & 0.06 \\
\hline & & $75-20$ & 0.02 & 2.2 & 1.5 & 7.8 & 0.01 \\
\hline \multirow{2}{*}{ Playas } & \multirow{2}{*}{17} & $30-70$ & 2.5 & 6.6 & 1.8 & 3.8 & 0.12 \\
\hline & & $70-120$ & 1.9 & 2.9 & 1.2 & 11.6 & 0.18 \\
\hline \multirow{2}{*}{ Sand Sheets } & \multirow{2}{*}{11} & $0-20$ & 8.7 & 7.1 & 3.9 & 0.9 & 0.09 \\
\hline & & $20-30$ & 2.6 & 1.3 & 0.9 & 1.7 & 0.08 \\
\hline \multirow{2}{*}{ Dry Wadis } & \multirow{2}{*}{15} & $35-80$ & 9.5 & 7.2 & 4.1 & 0.4 & 0.04 \\
\hline & & $80-120$ & 4.8 & 1.9 & 1.4 & 0.9 & 0.06 \\
\hline
\end{tabular}

Z: Zircon T: Tourmaline R: Rutile P: Pyroxenes A: Amphiboles B: Biotite. 


\section{REFERENCES}

Biscays, P. E. (1965). Mineralogy and sedimentation of recent deep sea clays in the Atlantic Ocean and adjacent seas and oceans. Geol. Soc. Am. Bull. 76: 803-832.

Brewer, R. (1964). Fabric and Mineral Analysis of Soils. Iohn Wiley and Sons Inc., New York

Brindly, G. W. and G. Brown (1980). Clay structure of clay minerals and their identification. Min. Soc. Monograph No. (5), London, UK. 495 pp.

Brough, P. A. (1986). Principles of Geographical Information Systems for Land Resources Assessment. Oxford University Press, 194p.

Brown, G. (1961). The X-ray Identification and Crystal Structures Minerals. SOC. Clay Mineral. Group. London

Burt, Rebecca Ed. (2004). Soil Survey Laboratory methods Manual. Soil Survey Investigations Report No. 42 Version 4.0 (NRCS)- USDA, Lincoln, Nebraska, USA.

Daels, L. (1986). Remote sensing fundamentals. Gent. State Univ., Gent., ITC. J., pp.1-19.

Dixon, J. B. and S. B. Weed (1977). Minerals in soil environments. Soil Sci., Soc. Am. Madison, Wisc., USA.

Dobos, E., E. Micheli, M.F. Baumgardner, L. Biehl and T. Helt (2000). Use of combined digital elevation model and satellite radiometric data for regional soil mapping. Geoderma. 97: 367-391.

EGSA "Egyptian Geological Survey Authority" (1988). "Egyptian General Petroleum Corporation: Geological Map of Egypt", Conoco Coral, printed in Germany by Institute fur Angewandte fGeodasie, Berlin, Technische Fachhochschule Berlin, Scale 1: 500000.

Folk, R. L. (1980). Petrology of Sedimentary Rock; Sylabus, Hamphill's publishing company, Austin, Texas. USA.

Gjems, O. (1967). Studies on clay minerals and clay mineral formation in soil profiles in Scondianvia. Jaurdunder Sokelsns Sertykk NR. 128: 305-407.

Green, K. (1992). Spatial imagery and GIS: integrated data for natural resource management. J. For. 90: 32-36.

Jackson, M. L. (1973). Clay minerals formations in soil genesis during the quaternary. Soil SCi., 99: 15

Milner, H. B. (1962). Sedimentary Petrology. George Allen and Unwin Ltd., Museum Street, London, U. K. V.1 and 11.

Sanad, S. (1972). "Geology and hydrology of Farafra Oasis, Western Desert, U.A.R. M. Sc. Fac. of., Assiut Univ

Stein, A. (1998). Integrating spatial statistics and remote sensing. INT. J., 19 (9): 1793-1814

Venkatarathnam, K. and W. B. Ryan (1971). Dispersal pattern of clay minerals in the sediments of the Eastern Mediterranean Sea Marine Geol., 11: 261-282.

Weaver, C. E. (1961). Clay mineralogy of late Cretaceous rocks in the Washaki Basin. Guide-book, Wyoming Geol. Ass. 16th Ann. Conf.: 148-154.

Zinck, J. A. (1988). Geomorphology and Soils. Internal Publ., ITC., Enschede, The Netherlands. 
الخصائص الجيومورفولوجية والأرضية والمعدنية لأراضي واحة الفرافرة

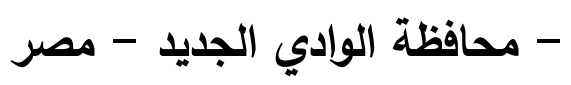

محمود سليمان محمد، يوسف قطب الغنيمى، شيماء كمال جنزور

معهة بحوث الأراضي والمياه والبيئة -مركز البحوث الزراعية

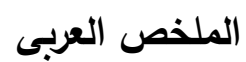

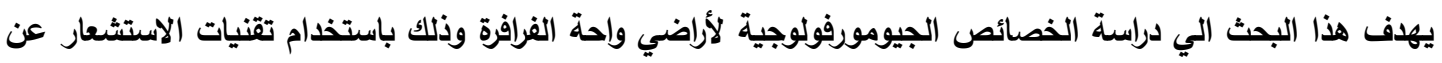

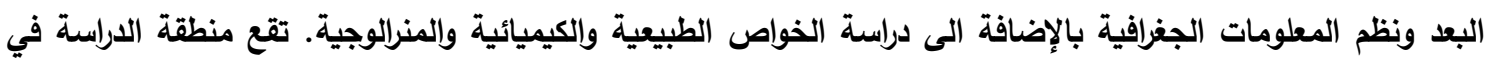

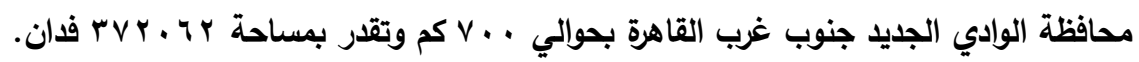

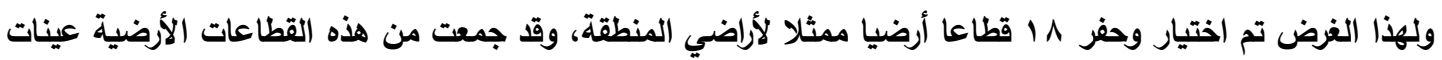

تمثل الاختلافات الرأسية لإجراء للتحليلات المعملية المختلفة.

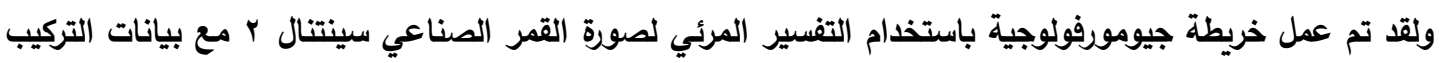

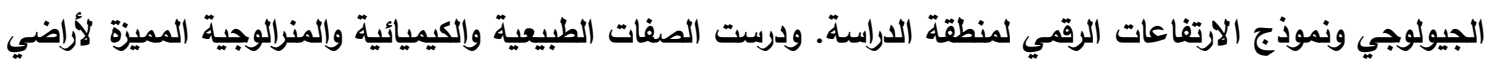

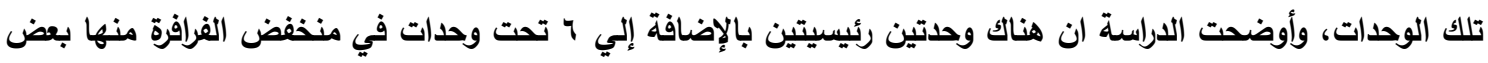

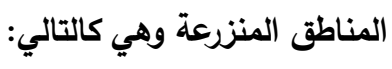
Chalky plain السهل الطبانشيرئ

Peni plain الثباه السهول الطبانيرول

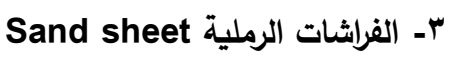

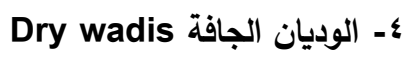
Pا البلايا Playa

Tocky Area أراضي صخرية البايا

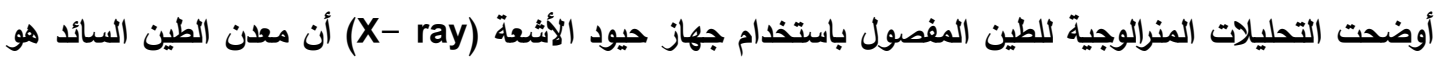

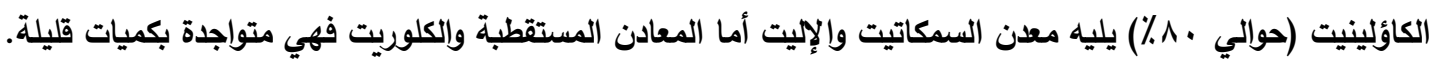

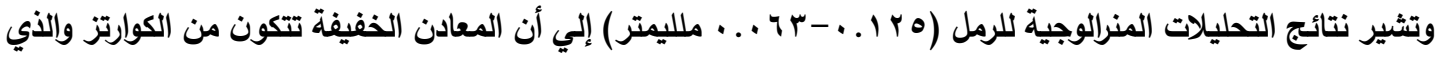

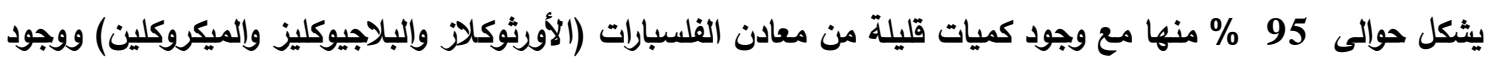

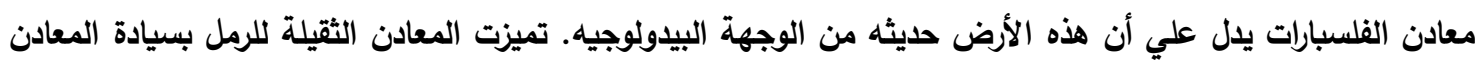

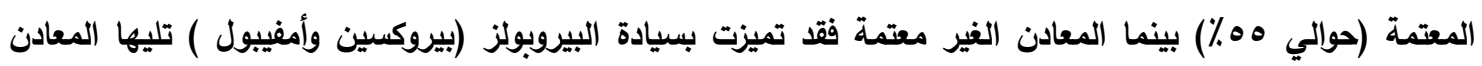

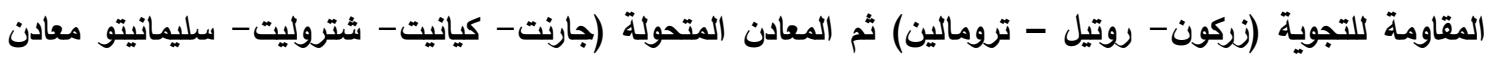

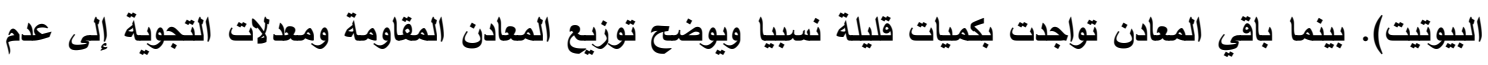

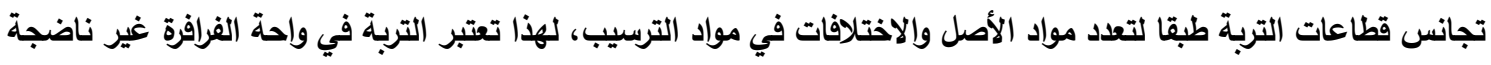
أو حايثة التكوين من الوجهة البيدولوجيه.

السادة المحكمين مركز البحوث الزراعية - الجيزة 